\title{
Chapter 17 \\ Physics and Philosophy of Physics in the Work of Mario Bunge
}

\author{
Gustavo E. Romero
}

\begin{abstract}
This brief review of Mario Bunge's research on physics begins with an analysis of his masterpiece Foundations of Physics, and then it discusses his other contributions to the philosophy of physics. Following that is a summary of his more recent reactions to scientific discoveries in physics and a discussion of his position about non-locality in quantum mechanics, as well as his changing opinions on the nature of spacetime. The paper ends with a brief assessment of Bunge's legacy concerning the foundations of physics.
\end{abstract}

\subsection{Introduction}

Before embarking on a more formal examination of Bunge's work in philosophy, physics and the overlap of both, I would like to indulge in some personal narrative and reflect on the depth of influence which Mario Bunge has had on my career, as an example of how he has been a central figure in the work of so many others over many decades. Given the purpose of this paper and the context of its inclusion in a larger work recognizing the breadth and depth of his footprint across many fields, such a diversion does not seem misplaced. Following this background story, there is a brief summary of some of the major points in Mario's own early life and academic career. This provides some context to the subsequent analysis of his works and his Cartesian approach to the development of a fundamental re-construction of the theory and practice of scientific methods.

Mario Bunge began academic studies in physics in March 1938. His doctoral thesis was presented at the University of La Plata in his native Argentina and was supervised by Guido Beck, a distinguished theoretical physicist (Bunge 1960). By 1945 Bunge had published papers on nuclear physics in Physical Review and Nature, two top international research journals. His involvement in philosophy and quixotic

\footnotetext{
G. E. Romero $(\square)$

Instituto Argentino de Radioastronomía (IAR, CCT La Plata, CONICET-CIC), Buenos Aires, Argentina
} 
enterprises such as the philosophical journal Minerva and the Argentine Workers University, of which he was founder and main driving force, resulted in long delays in his academic career. Bunge worked as a teaching assistant in physics courses at the University of La Plata until, after the fall of the Peronist government, he could obtain an appointment as Professor of Physics. Soon afterwards, he also gained positions in physics and philosophy at the University of Buenos Aires. From 1958 and until he left the country in 1963 he was Full Professor of Philosophy at the University of Buenos Aires. During this lengthy formative period Bunge assimilated research habits, was in direct contact with all major theories of modern physics and started to shape his unique philosophical views. His experiences as a research scientist and his involvement in the controversies in physics during this period, in particular those related to the interpretation of quantum mechanics, were key in the formulation of his philosophical project, which was later expressed in his Treatise on Basic Philosophy (1974-1989) and a series of books that followed. The basic outline of his philosophy was already present in his Foundations of Physics (Bunge 1967a), a superb work of clarification of all major theories in this field of science. This chapter discusses how physics and philosophy interrelate in Bunge's formative years and how this interrelation leads to a new approach to philosophy.

In his Other Inquisitions, the Argentine writer Jorge Luis Borges evokes a phrase (perhaps apocryphal) of William Henry Hudson, where Hudson says that many times in his life he undertook the study of metaphysics, but happiness always interrupted him. I confess, not without some melancholy, that few events have interrupted my many years of study and dedication to physics. However, I remember some bright moments of intellectual happiness in a life that may have lacked many things, but not thought. Even today I recall my excitement when I first read Mario Bunge's Foundations of Physics. It was my reading of Bunge's Foundations that reignited my enthusiasm for physics in the late 1980s. This was at a time when local and national politics were had more to do with petty and destructive power struggles than genuine leadership, and this had an adverse effect on researchers aspiring to further their knowledge unhindered by such negative external influences. His book had not been widely acknowledged by philosophers, but he did write extensively and with clarity about physics, using logic and mathematics. It was this rigorous approach which I adopted from the outset in my own pursuit of scientific knowledge.

When Bunge wrote about general relativity or quantum mechanics, he focused on the theories and their development: axiomatized, formalized, and clarified. Logic, mathematics, semantics: all were used in his analysis of a science in which I intended to base my own career. His work was a continuation of Hilbert's program, with knowledge of the formal limitations and carried out in the most rigorous possible way, by an expatriate Argentine who had studied in the same classrooms as me.

I decided to study all of Mario's work, and with my friend Santiago Pérez Bergliaffa we formed a group to read and analyze his books. By 1991 we had already mastered the essentials. It was then that we learned that Bunge was to teach a course on Epistemology at the University of Santa Catarina, in Florianópolis, Brazil. We did not hesitate: we bought a bus ticket (which we paid for with our lean wages) and 
went to hear Bunge. The course was brilliant and after posing many questions in class, we accepted his cordial invitation to the lobby of his hotel. He encouraged us to analyze the axiomatization of quantum mechanics. When we returned to Argentina, we decided to attack the problem. We discussed the technical issues with Mario, and later with our thesis supervisor. Shortly afterwards we had produced a new axiomatization of non-relativistic quantum mechanics which we sent to Bunge. To our surprise, he liked it and urged us to publish our results (something that had never occurred to us). Soon the paper appeared in the International Journal of Theoretical Physics (Perez Bergliaffa et al. 1993).

It would be the first of many articles I would write about philosophy of physics and scientific philosophy, despite having developed my main professional career in astrophysics. It would also be the beginning of a friendship with Mario that continues to the present day. When he presented his Between Two Worlds: Memoirs of a Philosopher-Scientist (Bunge 2016) at the University of Buenos Aires, I said publicly that my encounter with Mario Bunge has been one of the main events of my intellectual life. And of all his works, however much I have admired his Treatise (Bunge 1974a, b, 1977a, 1979, 1983a, b, c, 1985a, b, 1989), his Causality (Bunge 1959), his Chasing Reality (Bunge 2006), it was Foundations of Physics that had the greatest influence.

\subsection{Early Years and First Works on Physics and Its Philosophy}

Mario Bunge was born in West Florida, $17 \mathrm{~km}$ from the city of Buenos Aires, Argentina, on September 21, 1919. As the son of a doctor (Dr. Augusto Bunge) and socialist congressman of a patrician family, the young Mario always lived in an environment of intellectual freedom and social commitment. His mother of German origin, Marie Müser, had been a nurse with the Red Cross in China. Young Mario was soon attracted to philosophy. In 1936 he began to make more or less systematic readings of philosophical topics, but he soon became convinced that if he wanted to do philosophy seriously he had to first know science thoroughly.

$\mathrm{He}$ enrolled in the physics courses at the National University of La Plata in 1938, graduating in 1944, and later received his doctorate in 1952, with a thesis on relativistic electron kinematics. His mentor in physics was Guido Beck (19031988), who had been an assistant to Heisenberg in Leipzig.

In 1944 Bunge published his first physics article in the respected journal The Physical Review (Bunge 1944b) on the subject of the representation of nuclear forces. It was the first theoretical article on this subject undertaken in Argentina. In that same year he participated in the founding of the Argentine Physical Association (AFA, after its Spanish name, it is a society that still exists and is very active).

But before these scientific activities, Bunge had ventured into philosophy, publishing in 1939 a volume entitled Introdución al Estudio de los Grandes Pensadores 
(Introduction to the Study of Great Thinkers) (Bunge 1939). In 1944 he founded the philosophy magazine Minerva, where articles were published by several of the most respected thinkers in Latin America. Bunge himself wrote several papers for the magazine, which lasted just one year. One of those articles, "What is epistemology?" (Bunge 1944a), is perhaps the first article on modern philosophy of science written in Spanish.

In 1945, in Nature, he published a new article on nuclear physics, on the scattering cross-sections between protons and neutrons (Bunge 1945). In 1951 his first philosophy article in English appeared in the journal Science and Society: "What is chance?" (Bunge 1951). The article would be the starting point of Bunge's research on causality, which culminated in the publication of his classic book Causality (Bunge 1959). In that work, Bunge considered the role of causality in modern physics, and concluded that the world, although it is deterministic, is not strictly causal. Determinism implies legality in the sense that it should follow certain laws. There are no events that are not restricted by natural laws, but those laws are not necessarily causal. The world also admits probabilistic laws.

By that time (the mid-1950s), Bunge began to explore the different interpretations of quantum mechanics and to clarify his own position on this important and controversial issue. During this period, he published two important articles: A survey of the interpretations of quantum mechanics (Bunge 1956), and Strife about complementarity (Bunge 1955). That time also marks the first contact between Bunge and Karl Popper, who at the time also defended a realistic interpretation (although in his case a statistical one) of quantum mechanics. In the early 1960s Bunge moved first to the USA, then Germany, and finally settled in Canada in 1966, taking a chair in philosophy at McGill University, where he remained till his retirement and beyond.

Bunge produced a large number of works in philosophy of physics, published in various media such as the American Journal of Physics (Bunge 1956, 1957, 1961, 1966), The Monist (Bunge 1962), Reviews of Modern Physics (1967c), and British Journal for Philosophy of Science (1967d), among other journals. His essential vision of the great themes of physics would mature in these years and culminate in the publication, in 1967, of his extraordinary Foundations of Physics (op. cit.).

\subsection{Foundations of Physics}

Foundations of Physics (Bunge 1967a) was a pioneering and unique book. Pioneering for its rigorous style both from the philosophical and scientific points of view. Although many times physicists had written about philosophy, and philosophers had written on physics, this time the author was in complete control of both fields of research. It was a unique book also in its scope (it dealt with general methodology in philosophy of science and all the main theories of physics), in its depth, and in its rigor. Bunge presented rigorous axiomatizations of all the theories he discussed. 
The book is full of observations and clarifications of many conceptual errors that, given the clarity of Bunge's explanations, become obvious to the reader. It is a very stimulating text in that it provides many ideas and suggestions for further investigation. The second chapter includes an outline of a philosophical research program that Bunge went on to develop between 1974 and 1989: a complete system of scientific philosophy that includes semantics, ontology, epistemology and ethics. It provided the seed of what would later be the monumental Treatise on Basic Philosophy (op. cit.), a work of eight volumes in which the originality of Bunge's philosophical system is apparent. The Treatise may be the most important philosophical research project of the twentieth century and has its origin in the fact that, when conducting his research for the Foundations of Physics, Bunge realized that the underlying philosophy on which the science is based was not adequately developed. That gave rise to his development of a complete system of scientific philosophy, which would become a very influential reference.

After Foundations of Physics, Bunge continued to develop different aspects of his philosophy of physics in his Delaware Seminar (Bunge 1967e), Philosophy of Physics (Bunge 1973), Controversies in Physics (1983a, in Spanish), in Volume 7 of the Treatise, and in Mind and Matter (Bunge 2010). His most recent papers on philosophy of physics include: "Does the Aharonov-Bohm Effect Occur?" (Bunge 2015) and "Gravitational Waves and Spacetime" (Bunge 2018), both published in the journal Foundations of Science. Thus, Bunge has a continuous record of more than 70 years of work in both physics and philosophy of physics - a rare achievement. ${ }^{1}$

\subsection{Outline of Bunge's Views}

The two essential points of Bunge's philosophy of physics are its epistemological realism and ontological materialism. Scientific theories represent a reality that has an objective and independent existence apart from the subject. That does not imply, of course, that in our descriptions of reality we can dispense with a reference system. A description of reality can be perfectly objective and realistic, but it is always relative to a given reference frame.

In the 1960s, Bunge addressed the axiomatization of the major theories of physics, always highlighting the existence of a class of reference frames for each theory. These frames are concrete rather than conceptual, and certainly should not be confused with the coordinate systems we use to specify dimensional locations in physics. ${ }^{2}$ Bunge is very careful to point out that mathematics is a system of

\footnotetext{
${ }^{1}$ See his publications $(1967 b, 1967 \mathrm{~g}$ through to $1970 d$, and 1977c, 2000, 2002a, 2003, 2012) in the Reference list. And his publications with Kálnay (1969, 1983a, b) and with Maynez (1977).

${ }^{2}$ This confusion is so common among physicists that it can be found even in the young Einstein.
} 
formal abstractions that we use to represent aspects of real systems. Our ideas and representations of reality are mathematical, not reality itself.

Bunge represents the properties of physical systems by means of functions, or sets of functions, and concrete systems by sets. Thus, in general relativity for example, spacetime is represented by a real, 4-dimensional and pseudo-Riemannian manifold (a very particular class of set), and its properties by means of a set of 10 functions defined on the manifold. These functions are called the metric coefficients. The properties of material systems other than spacetime are represented by another set of functions structured in the so-called energy-impulse tensor. Then, the laws of the theory are represented by relations between functions that determine restrictions on the form of these through systems of equations. If the laws are purely local, the equations are differential. In general, the laws of physics are represented by integrodifferential equations, allowing for non-local actions.

In this way, the axiomatic structure of the theories is characterized by three sets of axioms: (1) the purely formal ones that fix the mathematical form of the theory, (2) the semantic axioms, which relate certain formal constructs with physical items, and (3) the law statements, which express the restrictions upon the state space of the referents of the theory. Law statements represent natural laws which are the patterns which arise in the occurrence of events in classes of things.

Bunge was the first to point out the importance of making explicit the semantic axioms in order to clarify the questions of interpretation of the theories of physics. To deal accurately with the interpretative aspects, he developed a whole semantic theory in the first two volumes of his Treatise.

Foundations of Physics is the most serious attempt that has been made so far to implement something similar to the Hilbert program of axiomatization of physics. The criticisms that some authors have made on the grounds that the program is obsolete due to Gödel's incompleteness theorems are baseless. These criticisms only show, in fact, a complete misunderstanding of the incompleteness theorems. The incompleteness theorems affirm that (1) no formal theory capable of describing arithmetic is at once consistent and complete, and (2) the consistency of a theory cannot be proved within the same theory. In the case of the theories of physics, if they are completely and correctly formalized, there is no danger of inconsistencies since the theories do not aspire to completeness: what we seek in physics is the best possible description of reality, not a theory complete in the formal sense. Nor are we interested in testing the consistency of our theories within the theory itself: every physicist works using appropriate meta-languages, even if he or she does not know it.

On the other hand, much is gained in clarity by axiomatizing a theory, as Bunge explains in detail in his Philosophy of Physics. For example, the axiomatization of quantum mechanics shows that the recurrent "observer" of textbooks is nowhere to be found. Quantum mechanics is not "subjective": the world does not exist, as Berkeley thought, because we observe it. It is the other way around: we can observe it because it exists. The observer plays no role in quantum mechanics. Bunge has pointed this out countless times, but even today we continue to hear about the 
supposed relationship between the consciousness of the observer and the "wave function", or worse, about its collapse.

The wave function of quantum mechanics does not "collapse". How would it collapse if it is a mathematical object, that is, a conceptual creation of the human mind? Countries, nerves, and buildings can collapse, but not the wave function. The wave function is a complex function in an infinite-dimensional space! Bunge showed with his axiomatic procedure that what actually evolves, in any case, is the quantum system in interaction with its environment, but not the wave function (Bunge 1967c, d, f).

The dynamic equation of quantum mechanics refers to physical systems, things such as electrons or photons. The wave function, as postulated by Born, simply gives the probability that the system is in this or that state, when the equation is solved for this or that set of boundary conditions. The relationship with the environment, Bunge correctly points out, must be non-linear, and cannot therefore be described by the wave function that satisfies a linear differential equation. To study the relationship between the quantum system and the environment, a quantum theory of measurement is needed. Long after Bunge wrote Foundations of Physics, the theory of decoherence was developed, which shows how quantum properties are rapidly lost when systems of many-particles interact with a complex environment.

\subsection{Bunge on Quantum Mechanics}

In his books and articles, Bunge has demolished many popular myths about quantum mechanics and its interpretations. He has pointed out, for example, that Heisenberg's inequalities are a theorem, not a principle (Bunge 1977b); and they have nothing to do with observations, since they are deduced from the noncommutativity of quantum operators and the (purely mathematical) inequality of Schwarz.

Bunge has pointed out how the wave function of Schrödinger's famous cat does not exist: what exists is a scribble that some stray people write in its place and to which they attribute supposed quantum properties. It is the same thing if they write $\left.\Psi\right|_{\text {cat }}$ as if they prefer $\left.\Psi\right|_{\text {Jupiter }}$ or $\left.\Psi\right|_{\text {sausage. }}$ The symbol $\Psi$ must denote a mathematical expression that satisfies the corresponding equation. In a system as complex as a cat, the quantum properties have disappeared at a level of composition just above that of a molecule. Hence, cats are not in a superposition of quantum states. Even if the master of the cat is Schrödinger!

In later works (for example, Controversies in Physics (Bunge 1983a) and Mind and Matter (Bunge 2010)) Bunge deals with the EPR paradox, Bell's inequalities, the alleged violation of realism by Aspect's experiments and the like. He also discussed the so-called quantum entanglement. Bunge always offers us the same thing: the most sensible response that corresponds to the real formalism of the theory. He points out, for example, that the refutation of Bell's inequalities does not imply that realism is not valid, but that deterministic theories with hidden variables 
are incompatible with the assumption of locality. Quantum mechanics is clearly not local. But that does not imply a problem for a realistic interpretation, unless you have a classicist conception of realism, something that certainly is not the case for Bunge.

Perhaps we could say that Bunge's interpretation is the most direct and simple of all the proposals. I call it the minimalist interpretation of quantum mechanics. We could say that it is a non-local realistic interpretation with a sui generis ontology, its famous "quanton" postulation. Bunge interprets the operators of quantum mechanics as mathematical objects that represent physical properties of the referents of the theory: these are sui generis quantum systems. These objects do not share the properties of classical systems. Under certain circumstances they can behave as if they were particles and under others as if they were waves, but they are neither waves nor particles, which are classic objects (Bunge 2002b). Much less they are both, particle and wave, at the same time. Quantons are quantum, singular objects, with quantum properties different from the classical ones. Spin, for example, is not the intrinsic angular momentum of the quantum system: quantum systems do not rotate. Spin resembles in some respects the intrinsic angular momentum of a classical object, but it is a quantum property. The same happens with the other properties, such as lepton number, isospin, location, velocity, and so forth. The only exception is energy, which is the capability of the system for changing. Energy, for Bunge, is the only truly universal property. It is what all existents share. In the quantum domain, it is a discrete property and, consequently, the quantons change discretely.

How is it possible that a composite quantum system, prepared in a certain state, remains in some way bound, even long after it has been separated, even if the separation is such that it makes any direct or causal interaction impossible? What is the origin of quantum entanglement? Before seeing how Bunge responds, certain facts, sometimes overlooked, need to be emphasized: (1) Entanglement is not universal (like gravitational action, say); it occurs only between quantum systems that were prepared in a certain way; (2) It does not involve the propagation of energy at a speed greater than that of light; (3) It does not imply a change of state of the system or a remote action; (4) It cannot be used to transmit information.

Let us examine an example of simple entanglement. Consider a non-polarized light source that emits photons in all directions. Suppose that two photons come out in opposite directions. Their total polarization, by the way they were produced, is null. Each of the photons, however, has an equal and opposite polarization. Quantum mechanics implies that one of them has a probability $1 / 2$ of having a polarization $(+)$ and a probability also $1 / 2$ of having the opposite polarization $(-) .{ }^{3}$ Suppose now that we determine that the polarization is $(-)$. Then, we conclude with probability 1 that the other photon has polarization $(+)$. However, the same theory of quantum mechanics predicts that if the other photon is an independent system, its probability of being in the $(+)$ mode is not 1 but $1 / 2$. The experiments show that the second photon is always in the state that corresponds to the initial preparation, regardless

\footnotetext{
${ }^{3}$ Photons have only two polarization modes.
} 
of the determination of the state of the first photon. The system is still "connected" in some way. This is the "spooky" action at distance mentioned by Einstein.

Actually, there is no connection, but non-local correlation: once an interlaced state has been formed, the system remains entangled regardless of the spatial separation of the components. Does it contradict common sense? Yes, for sure. And it is not the only thing that contradicts it in the quantum world. This should not surprise us, since common sense has been forged with experiences obtained in a different ontological level, the macroscopic one, which we call "classical".

When we specify the state of the first photon, the state of the second photon is specified, according to the initial preparation of the system. Once an interaction has destroyed the entanglement, the components are separated and there are no more correlations. Note that if we want to use entanglement to transmit information faster than light we will fail: there is no way that at the moment the second polarization is measured the value of the first one is known. That information can only be transmitted at the speed of light, as always. There is also no instant "work" on the second photon performed by the first. There is no change of state, but a specification of the state of the second photon that is correlated with that of the first photon. If there is work on the photon, the second detector does it, locally.

Bunge, then, invites us to accept the real world as it is: non-local, legal (the laws represented by the equations of quantum mechanics always apply), and independent of the cognitive subjects: it does not matter whether the second photon is registered by an instrument or interacts naturally. All these processes have been occurring in the universe long before human beings appeared on Earth, and they will continue to occur long after life has disappeared.

Bunge's position has sometimes been compared to that of Sir Karl Popper (see, for instance, Popper 1992). While it is true that Popper, like Bunge, was realistic in his interpretation of quantum mechanics, he thought that the wave function corresponds to a collection of particles and not to individual quantum objects. In that sense, Popper's position was similar to that of American physicist Leslie E. Ballentine, in his book Quantum Mechanics: A Modern Development (Ballantine 1998). Popper, on the other hand, was willing to accept that certain quantum phenomena have a relevance in the explanation of consciousness, something also unacceptable to Bunge. Popper supported indeterminism, in the sense that some non-legal events might occur. Bunge, on the contrary, always claimed that the world is fully legal, albeit in case of the quantum realm, the laws can be probabilistic. So, we can say that both thinkers agreed on the realistic interpretations of quantum mechanics, by differed on ontological views of many particular issues.

\subsection{Bunge on Fields}

Most of the Foundations is devoted to classical theories of physics, such as mechanics, electrodynamics, and the general theory of relativity. In Bunge's analysis of these theories, we can already glimpse the general ideas underlying what would 
later form the core of his ontology, expressed in volumes 3 and 4 of the Treatise. Bunge considers that the components of the world are corpuscles and fields. The form in which they associate or compose to form larger entities is different: fields overlap, while particles juxtapose (the classical ones at least).

Bunge's analysis of field theories is particularly illuminating. He clearly separates the mathematical from the physical concept of fields. A mathematical field is a conceptual object that satisfies a theory that incorporates a principle of minimum action and is described by Lagrangian density. A physical field, on the other hand, is a real object. Bunge distinguishes three types of theories of fields in physics: theories of pure, material and mixed fields. The first have as referents physical objects that cannot be eliminated by mere changes of the reference system adopted in the formulation of a model of the theory. The second type represent properties of extended material systems. Examples of pure fields are the electromagnetic and the gravitational fields. Examples of material fields are the velocity field of a fluid and the temperature field of a body. It is not possible to eliminate the electromagnetic or gravitational field globally by changing our description of nature. On the other hand, everything we say about material fields can be expressed in terms of particles, at least in principle. The theories of mixed fields are those that combine pure fields with material ones, such as macro-electromagnetism.

Bunge's analysis of the electromagnetic field is remarkably lucid. Among other issues, he points out that theory does not properly contain the hypothesis that charges are the sources of the field. Strictly speaking, electromagnetic theory is a theory of the interactions between fields and charged particles. The hypothesis that charges are sources of the field must be added to the Maxwell equations in order to discard the advanced contributions (determined by future events) to the solutions of the inhomogeneous Dalambertian equation. This is done by means of the application of the principle of causality. This hypothesis is logical, ontological and epistemologically independent from the rest of the theory.

Something similar happens with the general theory of relativity: the hypothesis that matter is the cause of the curvature of spacetime (gravitational field) is $a$ posteriori. Bunge points out other points poorly understood in connection with general relativity. For example, that the difference between gravitational and inertial mass is a mere conjecture, refuted by experience, and hence the identity of "both" masses is not a physical law. In fact, there are no such masses.

Bunge points out the purely heuristic character of the principle of equivalence (which can be derived as a theorem in his axiomatic analysis), and clarifies that the principle of general covariance is a meta-nomological statement (that is, a prescriptive statement for the formulation of basic laws). He also asserts the incompleteness of the theory, the fact that it is not only matter that determines the geometry of spacetime but matter plus the initial and contour conditions, and finally that the existence of gravitational waves is compatible with the theory, but it is not a direct theorem of it.

With the discovery of gravitational waves in 2016 (Abbott et al. 2016), Bunge modified his vision of the ontology of general relativity, admitting the identity of spacetime and the gravitational field (Bunge 2018). However, he has maintained his 
"presentist" position, in the sense that he thinks that only present events exist, and not future and the past events, contrary to what is held by "substantivalists" and most of the realists regarding spacetime, and my own controversy with him in this area (Romero 2012, 2013, 2015, 2017, 2018) points to an ongoing debate.

\subsection{Conclusion}

Throughout more than 70 years, Mario Bunge has examined and analyzed the foundations of physics. Unlike many other philosophers dedicated to the philosophy of this science, Bunge has also researched in physics and has been a university professor of physics. This has given him a unique insight and depth in his views on this field. Also, in a certain way, it has isolated him from his peers, more inclined to semantic discussions. Most of the philosophy that still appears in academic journals is based on examples that appeal to common sense or adopt a physics that is closer to that of Democritus than to modern science - Ladyman and Ross (2007) provide a critique on the current state of analytic philosophy.

Fifty years after its publication, Foundations of Physics continues to be a book of enormous depth. No one who engages with this text will leave empty-handed. I, on re-reading it for this paper, felt its extraordinary impact again, just like in those memorable days of my youth.

Acknowledgements I thank Mario Bunge for more than 25 years of stimulating discussions on physics and philosophy. I am also indebted to Santiago Perez Bergliaffa, Pablo Jacovkis, Daniela Pérez, Luciano Combi, Federico López Armengol, Janou Glaeser, and Héctor Vucetich for valuable discussions, questions, and criticisms. I am grateful with the members of the working group on Mario Bunge's work in Facebook, in particular with Gerardo Primero, Emerson Salinas Caparachin, Manuel Carroza Muro, Nicolás Pérez, Ed Cidd, Silvio Sánchez Mújica, Sergio Riva de Neyra, Gustavo Garay, and Matías Castro. I appreciate the kind hospitality of the University of Barcelona and the Institute of Cosmos Science (ICCUB) and its Head, Prof. Josep M. Paredes. Finally, I am thankful to Micheal Matthews for significantly improving the original manuscript and his superb job as editor of this volume. My research is supported by grant PIP 0338 (CONICET) and grant AYA2016-76012-C3-1P (Ministro de Educación, Cultura y Deporte, España).

\section{References}

Abbott, B. P., et al.. [LIGO Collaboration](2016). Observation of gravitational waves from a binary black hole merger. Physical Review Letters, 116, 061102.

Ballantine, L. E. (1998). Quantum mechanics: A modern development. Singapore: World Scientific.

Bunge, M. (1939). Introducción al estudio de los grandes pensadores. Conferencias, III, 105-109. and 124-126.

Bunge, M. (1944a). ¿Qué es la epistemología ? Minerva (Buenos Aires), 1, 27-43.

Bunge, M. (1944b). A new representation of types of nuclear forces. Physical Review, 65, 249.

Bunge, M. (1945). Neutron-proton scattering at 8.8 and $13 \mathrm{MeV}$. Nature, 156, 301.

Bunge, M. (1951). What is chance? Science and Society, 15, 209-231. 
Bunge, M. (1955). Strife about complementarity. British Journal for the Philosophy of Science, 6 , $1-12$. and 141-154.

Bunge, M. (1956). A survey of the interpretations of quantum mechanics. American Journal of Physics, 24, 272-286.

Bunge, M. (1957). Lagrangian formulation and mechanical interpretation. American Journal of Physics, 25, 211-218.

Bunge, M. (1959). Causality: The place of the causal principles in modern science. Cambridge, MA: Harvard University Press.

Bunge, M. (1960). Cinemática del electrón relativista. Tucumán: Universidad Nacional de Tucumán.

Bunge, M. (1961). Laws of physical laws. American Journal of Physics, 29, 518-529.

Bunge, M. (1962). Cosmology and magic. The Monist, 44, 116-141.

Bunge, M. (1966). Mach's critique of Newtonian mechanics. American Journal of Physics, 34, 585-596.

Bunge, M. (1967a). Foundations of physics. New York: Springer.

Bunge, M. (1967b). Scientific research. Berlin/Heidelberg/Nueva York: Springer.

Bunge, M. (1967c). Physical axiomatic. Reviews of Modern Physics, 39, 463-474.

Bunge, M. (1967d). Analogy in quantum mechanics: From insight to nonsense. British Journal for the Philosophy of Science, 18, 265-286.

Bunge, M. (1967e). Delaware seminar in the philosophy of science. Berlin/Heidelberg/Nueva York: Springer.

Bunge, M. (1967f). The structure and content of a physical theory. In M. Bunge (Ed.), Delaware seminar in the foundations of physics (pp. 15-27). Berlin/Heidelberg/New York: Springer.

Bunge, M. (1967g). Quanta y filosofía. Crítica, 1(3), 41-64.

Bunge, M. (1968). Physical time: The objective and relational theory. Philosophy of Science, 35, 355-388.

Bunge, M. (1969). Corrections to foundations of physics: Correct and incorrect. Synthese, 19, $443-$ 452.

Bunge, M. (1970a). Problems concerning inter-theory relations. In P. Weingartner \& G. Zecha (Eds.), Induction, physics and ethics (pp. 285-315). Dordrecht: Reidel.

Bunge, M. (1970b). The so-called fourth indeterminacy relation. Canadian Journal of Physics, 48, 1410-1411.

Bunge, M. (1970c). Virtual processes and virtual particles: Real or fictitious? International Journal of Theoretical Physics, 3(6), 507-508.

Bunge, M. (1970d). The arrow of time. International Journal of Theoretical Physics, 3(1), 77-83.

Bunge, M. (1973). Philosophy of physics. Dordrecht: Reidel.

Bunge, M. (1974a). Treatise on basic philosophy, vol. 1, sense and reference. Dordrecht/Boston: Reidel.

Bunge, M. (1974b). Treatise on basic philosophy, vol. 2, interpretation and truth. Dordrecht/Boston: Reidel.

Bunge, M. (1977a). Treatise on basic philosophy, vol. 3, the furniture of the world. Dordrecht: Reidel.

Bunge, M. (1977b). The interpretation of Heisenbergs inequalities. In H. Pfeiffer (Ed.), Denken und Umdenken: zu Werk und Wirkung von Werner Heisenberg (pp. 146-156). Munich: Piper.

Bunge, M. (1977c). Quantum mechanics and measurement. International Journal of Quantum Chemistry, 12(Suppl. 1), 1-13.

Bunge, M. (1979). Treatise on basic philosophy, vol. 4, a world of systems. Dordrecht/Boston: Reidel.

Bunge, M. (1983a). Controversias en Física. Madrid: Tecnos.

Bunge, M. (1983b). Treatise on basic philosophy, vol. 5, exploring the world. Dordrecht: Reidel.

Bunge, M. (1983c). Treatise on basic philosophy, vol. 6, understanding the world. Dordrecht: Reidel.

Bunge, M. (1985a). Treatise on basic philosophy, vol. 7, parte I, formal and physical sciences. Dordrecht: Reidel. 
Bunge, M. (1985b). Treatise on basic philosophy, vol. 7, parte II, life science, social science, and technology. Dordrecht: Reidel.

Bunge, M. (1989). Treatise on basic philosophy, vol. 8, the good and the right. Dordrecht/Boston: Reidel.

Bunge, M. (2000). Energy: Between physics and metaphysics. Science and Education, 9, 457-461.

Bunge, M. (2002a). Twenty-five centuries of quantum physics: From Pythagoras to us, and from subjectivism to realism. Science and Education, 12, 445-466.

Bunge, M. (2002b). Quantons are quaint but basic and real. Science and Education, 12, 587-597.

Bunge, M. (2003). Velocity operators and time energy relations in relativistic quantum mechanics. International Journal of Theoretical Physics, 42, 135-142.

Bunge, M. (2006). Chasing reality: Strife over realism. Toronto: Toronto University Press.

Bunge, M. (2010). Matter and mind (Boston Library in the Philosophy of Science, No. 287). Berlin: Springer.

Bunge, M. (2012). Does quantum physics refute realism, materialism and determinism? Science and Education, 21, 1601-1610.

Bunge, M. (2015). Does the Aharonov-Bohm effect occur? Foundations of Science, 20, 129-133.

Bunge, M. (2016). Between two worlds: Memoirs of a philosopher-scientist. Berlin: Springer.

Bunge, M. (2018). Gravitational waves and spacetime. Foundations of Science, 23(2), 405-409.

Bunge, M., \& García Maynez, A. (1977). A relational theory of physical space. International Journal Theoretical Physics, 15, 961-972.

Bunge, M., \& Kálnay, A. J. (1969). A covariant position operator for the relativistic electron. Progress of Theoretical Physics, 42, 1445-1459.

Bunge, M., \& Kálnay, A. J. (1983a). Solution to two paradoxes in the quantum theory of unstable systems. Nuovo Cimento, B77, 1-9.

Bunge, M., \& Kálnay, A. J. (1983b). Real successive measurements on unstable quantum systems take nonvanishing time intervals and do not prevent them from decaying. Nuovo Cimento, B77, $10-18$.

Ladyman, J., \& Ross, D. (2007). Everything must go. Oxford: Oxford University Press.

Perez Bergliaffa, S. E., Romero, G. E., \& Vucetich, H. (1993). Axiomatic foundations of nonrelativistic quantum mechanics: A realistic approach. International Journal of Theoretical Physics, 32, 1507-1522.

Popper, K. (1992). Quantum theory and the schism in physics: From the postscript to the logic of scientific discovery. London/New York: Routledge.

Romero, G. E. (2012). Parmenides reloaded. Foundations of Science, 17, 291-299.

Romero, G. E. (2013). From change to spacetime: An Eleatic journey. Foundations of Science, 18, 139-148.

Romero, G. E. (2015). Present time. Foundations of Science, 20, 135-145.

Romero, G. E. (2017). On the ontology of spacetime: Substantivalism, relationism, eternalism, and emergence. Foundations of Science, 22, 141-159.

Romero, G. E. (2018). Mario Bunge on gravitational waves and the existence of spacetime. Foundations of Science, 23(2), 405-409. 